Cahiers Société

\title{
Castoriadis et les trois voies de la socio-ontologie
}

\section{Danilo Martuccelli}

Numéro 1, mai 2019

Castoriadis et les sciences sociales

URI : https://id.erudit.org/iderudit/1068421ar

DOI : https://doi.org/10.7202/1068421ar

Aller au sommaire du numéro

Éditeur(s)

Collectif Société

ISSN

2562-5373 (imprimé)

2562-5381 (numérique)

Découvrir la revue

Citer cet article

Martuccelli, D. (2019). Castoriadis et les trois voies de la socio-ontologie. Cahiers Société, (1), 63-90. https://doi.org/10.7202/1068421ar

\section{Résumé de l'article}

L'article propose une interprétation de l'oeuvre de Castoriadis à partir de considérations socio-ontologiques. Après une rapide évocation de la principale fonction que l'ontologie a eue à la naissance de la sociologie, l'article se centre sur ce qui est devenu, au moins implicitement, le commun dénominateur des questions ontologiques dans la sociologie - le fait que la réalité est ce que résiste. Cette problématique est au coeur de trois grandes voies socio-ontologiques - la représentation, la production et l'action - qui, en dépit des emprunts réciproques, n'en tracent pas moins des problématisations indépendantes. L'intérêt de l'oeuvre de Castoriadis est d'avoir proposé plus ou moins explicitement une socio-ontologie totale autour de l'imaginaire qui, cependant, comme l'article s'efforce de montrer, n'est finalement qu'une variante de l'ontologie de la représentation. 


\title{
Castoriadis et les trois voies de la socio-ontologie
}

\author{
Danilo MARTUCCELLI \\ Universidad Diego Portales et Université Paris Descartes
}

Cet article propose une relecture critique de l'œuvre de Cornelius Castoriadis à partir de questions proprement ontologiques. Il est animé par une double vocation. D'une part, il vise à « insérer » Castoriadis dans les débats contemporains de la théorie sociale autour des questions ontologiques, en montrant, au-delà de son indéniable originalité, la manière dont il peut (et doit) être lu en référence à d'autres perspectives proches. D'autre part, et cette fois-ci au-delà de l'œuvre même de Castoriadis, l'article explore quelques-uns des nouveaux enjeux qui sont en train de s'ouvrir au fur et à mesure que se complexifie l'étude des relations entre la sociologie et l'ontologie.

La séduction indéniable de l'œuvre de Castoriadis procède du fait qu'il a, au moins implicitement, proposé une ontologie totale visant à articuler ce qui, le plus souvent, a été développé de manière indépendante : des ontologies de la représentation, de la production et de l'action. À l'encontre de cette division, Castoriadis a proposé une ontologie totale autour de l'imaginaire. Or, comme nous le verrons, malgré les ouvertures et sa volonté explicite d'intégrer dans son ontologie du monde social le travail («production ») ou la praxis («action»), son ontologie de l'institution imaginaire de la société reste finalement ancrée autour du modèle de la représentation.

Nous procéderons en cinq grandes étapes. Nous commencerons par un bref rappel des manières dont la question ontologique a été posée, dès ses débuts, dans la sociologie, afin d'asseoir la spécificité de la vie sociale vis-à-vis d'autres domaines ontologiques. Puis, nous nous centrerons de manière plus approfondie sur les trois grandes perspectives de problématisation socio-ontologique de la réalité autour de la représentation, de la production et de l'action'. Ce n'est qu'une fois ces développements effectués que nous aborderons de façon critique dans la dernière partie les promesses et les problèmes de l'ontologie de l'imaginaire social propre à Castoriadis.

\footnotetext{
${ }^{1}$ Notre but n'est nullement de parvenir à une impossible présentation exhaustive de ces démarches, mais de dégager de la façon la plus explicite possible, en conversation critique avec l'œuvre de Castoriadis, chacune de ces épures. Nous n'évoquerons donc, dans ce qui suit, que quelques travaux particulièrement significatifs et laisserons en dehors de cet article bien des travaux qui pourraient y trouver une place.
} 


\section{Ontologie et sociologie}

Si la question ontologique est constitutive du regard philosophique sur le monde, il en va au fond quelque peu de même pour les réflexions sociologiques où l'ontologie du social fait partie prenante de la discipline depuis sa naissance. Cependant, à ses débuts, les considérations proprement ontologiques furent rapidement subordonnées à des préoccupations épistémologiques. En effet, l'intérêt sociologique pour l'ontologie était dicté par la question du type d'entités susceptibles d'entrer dans une explication. Comme le résume Berthelot, dans les grandes traditions intellectuelles, que ce soit chez Durkheim ou chez Weber, «l'ontologie reste arrimée à des intérêts épistémologiques (de fiabilité et de pertinence de la connaissance) et ne dépasse pas la postulation initiale de propriétés génériques revendiquées ou exclues ${ }^{2} »$. Rien n'atteste mieux de cet «arrimage » que la célèbre distinction ontologique entre les sciences de la nature et les sciences de l'esprit, induisant une division épistémologique (« explication »vs « compréhension»), plus ou moins radicale, entre ces disciplines ; ou à l'inverse, les positions qui, à partir d'une vision moniste - le monde naturel et le monde social seraient soumis à une même ontologie - défendent la nécessité d'une épistémologie commune entre disciplines³.

Ces distinctions sont importantes, et ont engagé des réflexions théoriques majeures, notamment à l'heure de différencier la sociologie d'autres disciplines, mais elles ont peu animé, sinon sous une forme proprement épistémologique, des études sur la vie sociale. Or, à côté de ces réflexions " premières » sur le monde social, il est possible de repérer un autre style d'analyse ontologique. Sa spécificité s'éclaircira progressivement, mais disons qu'à la différence des études précédentes, la question principale n'est pas de dire «ce qu'est le réel» en définissant les éléments premiers du monde social, mais plutôt d'interroger les problèmes que certaines questions ontologiques posent à la pratique sociologique. Afin de distinguer cette famille des problématisations sociologiques des questions ontologiques, nous parlerons de démarches socio-ontologiques. C'est dans ce cadre que nous analyserons les propositions de Castoriadis.

Un point est commun à toutes les socio-ontologies : le postulat que la réalité est ce qui résiste. Impossible de négliger cette dimension : il y va depuis Durkheim de la

\footnotetext{
2 Jean-Michel Berthelot, "Sociologie et ontologie », dans L'enquête ontologique, dir. Pierre Livet et Ruwen Ogien, Paris, Éditions de l'EHESS, 2000, p. 76. Pourtant, y compris dans ce cadre, il a déjà été question de la relative indépendance des affaires ontologiques et épistémologiques, comme chez Durkheim qui défend à la fois une double ontologie (nature et société) tout en se prononçant pour un monisme épistémologique.

3 Certaines conceptions ontologiques contemporaines privilégient une vision stratifiée de la réalité (physico-chimique, biologique, psychologique...) faisant de la notion d'émergence un des axes majeurs de leur conception du monde (comme l'émergence de la vie à partir de la matière, ou l'esprit de la vie). Cf. Daniel Andler, Anne Fagot-Largeault, Bertrand Saint-Sernin, Philosophie des sciences, Paris, Gallimard, 2002, 2 vol. ; Frédéric Nef, Traité d'ontologie, Paris, Gallimard, 2009.
} 
caractérisation même des faits sociaux - des phénomènes externes à l'individu et caractérisés par leur objectivité, leur extériorité et leur sanction4. Si ces trois aspects sont parfois discutables (chez Durkheim la réalité apparaît tantôt comme une extériorité matérielle évidente, tantôt elle est assimilée - et réduite - à l'objectivité d'un collectif), ils n'en définissent pas moins le propre de la réalité sociale au travers de coercitions qui seraient particulières à la vie sociale. Certes, l'idée que les faits sociaux, en eux-mêmes, et indépendamment des croyances et des actions individuelles, puissent exercer des contraintes sur les acteurs est une proposition polémique. Et pourtant, en dépit de cette hypostase en partie illégitime, il y a quelque chose de foncièrement juste dans la vision durkheimienne, lorsqu'elle souligne l' «extériorité » des contraintes que la vie sociale est susceptible d'opposer aux acteurs. En tout cas, si on abandonne l'idée que les faits sociaux existent indépendamment des croyances et des actions individuelles, et qu'ils exercent des contraintes sur les individus, on aura de la peine à défendre l'idée d'une science sociale autonome5.

Cependant, et pour étrange que cela puisse paraître, les coercitions, comme manifestations principales de la résistance de la réalité, ont souvent été mobilisées de manière fort imprécise par les sociologues. Par «contrainte », Durkheim en effet désignait tour à tour les obstacles que le monde externe impose aux acteurs dans la poursuite de leurs objectifs, les divers effets de l'environnement social (notamment de nature morphologique) sur les actions, des contraintes liées à la socialisation, ou encore, dans une liste non exhaustive, la force des règles morales ou de l'autorité, et les sanctions qu'un groupe social est capable d'imposer aux membres qui ne les respecteraient pas. En fait, lorsque Durkheim parle de "contraintes ", il pense fréquemment «influence», et même influence légitime, lorsque par exemple la «contrainte» de l'autorité est reconnue et acceptée par les individus. En tout cas, c'est cette famille de contraintes qui l'intéressera de plus en plus au cours de sa vie intellectuelle. Depuis ses travaux, il est fréquent de différencier entre divers types de contraintes 6 .

Pourtant, et c'est ce qu'il nous intéresse de souligner ici, la différenciation établie entre les diverses coercitions s'estompe devant une grande similitude fonctionnelle : toutes les contraintes sont perçues de façon analogue, toutes imposent une limite plus ou moins indépassable et évidente aux acteurs. Or, cette attitude laisse entier le problème concret $\mathrm{du}$ travail des contraintes. Le «social» devient un niveau d'agencement particulier de la réalité, soumis aux conditionnements de "courants », de «forces », de «contraintes" dont le travail effectif au niveau de l'action reste

\footnotetext{
4 Émile Durkheim, Les règles de la méthode sociologique, Paris, PUF, 1987 [1895].

5 Ruwen Ogien, «Philosophie des sciences sociales », dans Épistémologie des sciences sociales, dir. Jean-Michel Berthelot, Paris, PUF, 2001, p. 535.

6 Par exemple, Giddens différencie entre contraintes matérielles (données par le monde matériel et la qualité physique des corps) ou structurelles (dépendant des contextes d'action), ou encore des sanctions négatives (des contraintes qui résultent des activités punitives entre acteurs). $C f$. Anthony Giddens, $L a$ constitution de la société, Paris, PUF, 1987 [1984].
} 
souvent « opaque ». La question des coercitions n'abandonne jamais la sociologie, mais devient essentiellement une affaire d'inférence logique, de récits d'acteurs ou de statistiques. Pourquoi cette réduction ? Parce que, comme on le verra, l'essentiel de la sociologie - à commencer par Durkheim - s'est produit en privilégiant l'ontologie de la représentation. Or, si cette manière de conceptualiser les contraintes est ô combien légitime, elle n'épuise certainement pas les manières dont les sciences sociales peuvent et doivent problématiser la question de la réalité7.

D’une manière schématique, et sans exhaustivité, il est en effet possible de différencier trois grandes perspectives socio-ontologiques: représentation, production et action $^{8}$. Si certains aspects leur sont communs (à commencer par le postulat de base : la réalité est ce qui résiste), ils ne possèdent pas moins une personnalité analytique indiscutable : chaque fois, ce sont des questions différentes qui sont posées ; chaque fois, ce sont des problèmes distincts qu'il s'agit de résoudre. Bien entendu, il est fréquent qu'un seul auteur - Castoriadis en est un exemple paradigmatique - inscrive ses travaux dans différentes perspectives ou qu'une seule tradition intellectuelle (le constructivisme, le marxisme, le pragmatisme...) mobilise des réflexions ontologiques à la charnière des différentes épures. Pourtant, et malgré ces emprunts et croisements, il n'en reste pas moins que chaque épure non seulement définit une problématisation différente, mais qu'elle suscite surtout des controverses intellectuelles diverses autour de la réalité et de ses résistances.

\footnotetext{
7 Le primat de la traduction épistémologique des questions « ontologiques » est bien visible dans les débats autour de la distinction entre l'explication et la compréhension, sciences nomothétiques ou idéographiques, afin de délimiter un domaine spécifique aux sciences sociales les différenciant des sciences naturelles. Ni cet objectif ni ce souci n'ont disparu, et il serait possible d'analyser bien des questions ontologiques actuelles (autour des sciences cognitives, de la psychologie évolutive, de la sociobiologie, des conceptions stratifiées de la réalité...) dans le sillage de cette modalité de problématisation.

8 Dans les dernières décennies, une nouvelle vision onto-sociologique s'organise autour de la circulation - et dans ces études, l'influence de Deleuze et Guattari est souvent patente (et la reprise des mots comme flux, plis, rhizomes, déterritorialisations...). En dépit de certains excès métaphoriques, ces démarches soulignent la diversité des flux en circulation (Scott Lash et John Urry, Economies of Signs and Space, London, Sage, 1994); la resignification des objets lors de leurs déplacements (et donc à terme sur leur métamorphose ontologique) ou les différents scapes qui organisent leur circulation dans la globalisation (Arjun Appadurai (dir.), The Social Life of Things, Cambridge, Cambridge University Press, 1986 et Modernity at Large, Minneapolis and London, University of Minnesota Press, 1996). D'autres encore, ou les mêmes à d'autres moments de leurs trajectoires professionnelles, proposent même de remplacer l'idée de société - et l'idée d'ordre qui lui est spécifique - par la notion de mobilité (John Urry, Sociology beyond Societies, London, Routledge, 2000 et Global Complexity, Cambridge, Polity Press, 2003). Toujours au sein de cette vision, on parlera même d'un «troisième-espace » en tant que nouvelle matrice ontologique de connexion entre les flux et les espaces (Edward W. Soja, Thirdspace, Oxford, Blackwell, 1996).
} 


\section{Réalité et représentation}

La problématisation de la réalité comme représentation constitue une des plus vénérables questions de la philosophie. Pourtant, cela n'a pas empêché la sociologie de développer un regard quelque peu spécifique autour du constructivisme9. Les variantes sont multiples, mais l'idée maîtresse, au fond, est souvent la même. Animal symbolique, l'individu a la capacité originaire à installer un monde spécifique, familier et protecteur - transitionnel, dira Donald Winnicott - autour de luii ${ }^{10}$. Sa relation à la réalité est toujours enveloppée par un ensemble de médiations culturelles ${ }^{11}$. La vie en société se déroule toujours au milieu et au travers de catégories et de significations, même s'il est possible de différencier entre d'un côté, un terreau premier, toujours déjà-là, « objectif », souvent en lien avec un environnement " naturel », et de l'autre côté, ce qui est justement spécifique au « social », une sorte d' "étage » supplémentaire et construit de significations ${ }^{12}$. D'ailleurs, la célèbre notion de réel chez Lacan s'inscrit dans cette distinction : le réel ce n'est pas la réalité, au contraire, le réel est ce qui échappe au saisissement symbolique, un au-delà (un non ou hors symbolisable) qui nous saisit en dehors de toute signification ${ }^{13}$. Et c'est dans cette même lignée qu'on a souvent différencié entre les traits « objectifs» de la nature et les formes « culturelles » qu'elle peut prendre dans différentes sociétés ${ }^{14}$ ou plus largement que la distinction entre le naturel et le culturel, sans être arbitraire, est évaluée comme largement spécifique aux différents systèmes culturels 15 .

En tout cas, hormis quelques positions extrêmes, peu de démarches constructivistes nient au fond l'existence d'un « réel » déjà-là ${ }^{16}$. En revanche, elles soulignent

\footnotetext{
9 Le constructivisme est à la mode depuis quelques années, mais la véritable source de cette démarche revient à Kant ( $c f$. Emmanuel Kant, Critique de la raison pure, Paris, Flammarion, 2006 [1787]). C'est lui qui, pour la première fois, affirme que chaque fois que les individus décrivent le monde, leurs descriptions sont modelées par des concepts - ce qui rend possible justement la description du monde à partir de perspectives différentes. Certes, il peut sembler excessif de faire remonter l'origine du constructivisme à Kant, pourtant, sa démarche ouvre bien la voie à l'idée que nous ne saurons jamais ce « qu'est» la chose en soi, et que tout ce que nous pouvons faire est de décrire le monde à partir de nos « langages ». Il s'agit d'ailleurs d'une dimension de son œuvre fort bien soulignée et prolongée par Ernst Cassirer, Philosophie des formes symboliques, Paris, Minuit, 1972 [1953-1957], 3 vol.

10 Donald W. Winnicott, Jeu et réalité, Paris, Gallimard, 1981 [1971].

11 Clifford Geertz, The interpretation of cultures, New York, Basic Books, 1973.

12 Paul Watzlawick, La réalité de la réalité, Paris, Seuil, 1978 [1976].

13 Récemment, Boltanski a introduit une variante en distinguant entre le "monde », où émergerait tout ce qui arrive de façon sporadique et ontologiquement non maîtrisée ; le « réel » attaché à des manifestations circonstancielles et singulières ; et enfin, la « réalité » qui s'appuierait sur une sélection et organisation des possibilités qu'offre le monde, autant dire un ensemble de régularités. $C f$. Luc Boltanski, De la critique, Paris, Gallimard, 2009 et Énigmes et complots, Paris, Gallimard, 2012.

14 Serge Moscovici, Essai sur l'histoire humaine de la nature, Paris, Flammarion, 1977.

15 Philippe Descola, Par-delà nature et culture, Paris, Gallimard, 2005.

16 Le malentendu est pourtant souvent de rigueur à propos du «constructivisme radical ", un courant qui, depuis les années 1950, a mis en question la distinction «évidente » entre le sujet et l'objet (entre ce qui est perçu et ce qui a bien lieu dans la réalité). La connaissance se fait toujours à partir d'une
} 
fortement le fait que le réel est susceptible de recevoir un nombre pluriel de traductions culturelles - sous forme justement de « réalité ». La discussion porte donc sur l'étendue et la nature des traductions possibles du « réel » en " réalité », c'est-à-dire, du passage de l'expérience (ou de l'hypothèse) de l'existence d'un domaine intransitif indépendamment des individus - le réel - à sa mise en forme culturelle et surtout institutionnelle en tant que réalité. L'important est de cerner la "réalité » sociale au travers des « représentations ».

Tout en s'insérant dans une interrogation proprement philosophique ${ }^{17}$, la perspective socio-ontologique de la représentation a une indubitable spécificité - elle souligne notamment le rôle décisif des institutions dans la construction sociale de la réalité. C'est par ce truchement que la sociologie a repris et retravaillé la relation entre les mots et les choses. Si l'individu comme animal symbolique possède la capacité originaire de «mettre » un monde entre lui et la nature, ce sont les soubassements institutionnels de la construction sociale du monde qui retiennent l'attention des sociologues. Comme cela est déjà visible dans l'étude pionnière de Durkheim sur la religion, ce sont alors les manières dont les réalités sociales informent les catégories cognitives qui ont fait l'objet de leur attention - le but étant d'analyser tout autant la genèse sociale des concepts que le travail de maintien du monde social qu'ils permettent ${ }^{18}$. Cette préoccupation est toujours active comme le montrent, par exemple, l'étude de Bourdieu sur l'État et son rôle décisif dans la définition légitime de la réalité dans les temps modernes ${ }^{19}$ ou Françoise Héritier qui, en revenant sur « les éléments du réel immuables, récurrents, qu'il n'est pas possible de décortiquer », souligne le rôle fondateur du partage mâle/femelle dans la constitution du « critère de l'identique et du différent ${ }^{20} »$.

Notons-le : si cette posture n'est pas exclusive aux démarches socio-ontologiques, elle souligne avec une grande force le fait que la vie en société se déroule au travers de catégories qui, non seulement rendent compte de la réalité, mais à proprement

expérience d'observation et dans ce sens, la réalité est toujours appréhendée à partir du champ expérientiel d'un observateur. Autant dire que la connaissance sur le monde est une connaissance sur l'observation de l'observateur (une observation de second degré) et par voie de conséquence que toute observation fait elle-même partie du monde qu'elle observe. En sociologie, c'est Luhmann qui incarne le mieux cette orientation indissociablement épistémologique et ontologique à travers un « constructivisme opérationnel » conséquent - le constructivisme dû stricto sensu aux représentations s'ouvre alors vers un constructivisme ontologique d'un autre type ( $C f$. Niklas Luhmann, Systèmes sociaux, Québec, Presses de l'Université de Laval, 2010 [1984]). Nous y reviendrons dans le prochain paragraphe.

17 Cette problématique apparaît comme un avatar de la querelle des universaux : cette question lancinante de la philosophie du Moyen Âge qui, pendant des siècles, a fait référence à la discussion pour savoir si la réalité se réduit à sa désignation (nominalistes) ou si, au contraire, elle transcende ses désignations (réalistes).

18 Émile Durkheim, Les formes élémentaires de la vie religieuse, Paris, PUF, 1985 [1912].

19 Pierre Bourdieu, Sur l'État. Cours au Collège de France, 1989-1992, Paris, Seuil, 2012.

${ }^{20}$ Françoise Héritier, Masculin/Féminin, Paris, Odile Jacob, 1996 et «Les racines corporelles de la pensée », Le Débat, n 159, 2010, p. 111-127. 
parler, la structurent justement comme réalité. Comme nous le verrons ci-dessous, c'est dans ce cadre - par la notion d'imaginaire - que se place le noyau dur de la vision socio-ontologique de Castoriadis. La réalité sociale, abordée par le paradigme de la représentation, est donc indissociable d'un travail permanent de mise en cohérence symbolique ${ }^{21}$. Dans ces démarches, la représentation s'ouvre donc au travail des institutions du sens et à la question de l'ordre social22. Puisque la vie sociale est constamment une réalité perçue partagée, l'essentiel est de comprendre comment cette condition est continuellement renouvelée. L'extériorisation de la vie sociale est abordée par une typification d'habitudes et de routines donnant lieu progressivement à une institutionnalisation des conduites et à la formation d'un ensemble de rôles ou d'habitus. Variables selon les sociétés et les périodes, les institutions ont un effet de contrôle important puisqu'elles définissent des modèles prédéfinis de conduite canalisant dans un sens déterminé les actions. En bref, c'est bel et bien du travail de maintien des représentations par les institutions, plutôt que de la simple mise en langage du monde dont il est question dans les socio-ontologies de la représentation (ce qui intègre d'ailleurs les manières dont se construisent et s'instituent les catégories cognitives de la vie sociale 23 , mais aussi les façons dont le structuralisme « constructiviste » des épistémès dominantes rend compte de l'évolution des connaissances collectives ${ }^{24}$ ).

Le travail institutionnel est d'autant plus souligné que les démarches socioontologiques sont fort sensibles à la "nature » même des médiations ou des dispositifs symboliques; au fait qu'ils constituent une "sphère » à part, dotée d'un fonctionnement interne spécifique, se différenciant tout autant de la réalité matérielle que de la dimension proprement subjective ou cognitive 25 . Pourtant, et derrière cet accord de principe quant à la « réalité » de cette sphère, les désaccords font rage à l'heure de concevoir la "nature » de ces médiations (signes, symboles, significations, monde vécu, frontières, scripts, culture, imaginaire bien sûr...) ou les façons de rendre compte de l'usage qu'en font les acteurs (sens visé, communications, cadrages, échanges...). La prise en compte de cette pluralité de représentations est même à la base des travaux sur la déconstruction, sur l'hégémonie, voire sur les processus par lesquels certaines questions sociales deviennent - ou non - objet des représentations collectives ou médiatiques. Mais cette pluralité est aussi à la base du travail critique sur l'étiquetage social et les violences symboliques, qu'il s'agisse des dénominations (délinquants, déviants, minorités...) ou des catégories statistiques ; dans tous les cas, c'est le processus d'institutionnalisation, et par ce truchement de construction sociale

\footnotetext{
${ }^{21}$ Peter Berger et Thomas Luckmann, La construction sociale de la réalité, Paris, Armand Colin, 2006 [1966].

22 Vincent Descombes, Les institutions du sens, Paris, Minuit, 1996.

23 Aaron Cicourel, Cognitive Sociology, Harmondsworth, Penguin, 1974.

24 Michel Foucault, Archéologie du savoir, Paris, Gallimard, 1969.

25 Margaret R. Somers, «What's political or cultural about the political culture concept ? Toward an historical sociology of concept formation », Sociological Theory, vol. 13, n 2, 1995, p. 113-144.
} 
de la réalité, qui retient l'attention ${ }^{26}$. Même lorsque les dimensions "matérielles » sont davantage prises en compte, qu'il s'agisse des identités culturelles ${ }^{27}$, des catégories statistiques ou socioprofessionnelles ${ }^{28}$, des luttes sociales ${ }^{29}$, du sexe ou de genreso, l'objectif principal est toujours de rappeler les manières dont chacune de ces réalités, et au-delà d'elles le monde social tout court, est constitué par des « représentations ».

Plus récemment, c'est aussi dans ce cadre qu'il faut interpréter les études qui, notamment aux États-Unis, renouvellent l'analyse des manières dont la culture informe l'action. Du rôle et du primat traditionnellement accordés aux « contextes », on passe aux «textes » : à savoir, la fonction spécifique qui revient à la culture et aux déterminants narratifs dans la structuration de l'action, que ce soit au niveau des scripts sexuels, des scénarii politiques ou des storytellings commerciaux ${ }^{31}$. La construction des situations et des conduites se doit alors de privilégier, et d'aborder avec plus de minutie que par le passé, la fonction exacte que les différentes formes culturelles valeurs, cadres, répertoires, scripts, récits, frontières morales, institutions... - détiennent dans la mise en œuvre de la vie sociale. La reconnaissance de la diversité et de l'hétérogénéité des représentations invite à analyser le travail d'imposition des catégories symboliques communes - en fait dominantes - du monde.

Dans les démarches socio-ontologiques, il est aussi question des usages stratégiques des représentations. C'est un aspect qui a été particulièrement bien abordé par Goffman32. En interrogeant la "nature» de la vie sociale, il s'est surtout intéressé aux diverses manières dont il est possible d'imposer un cadre (de représentation) à une expérience. À la différence alors de positions radicales mettant en cause la réalité en tant que telle, ou s'interrogeant sur les entités premières, Goffman s'intéresse aux multiples relations que les acteurs entretiennent avec la réalité. La "réalité » devient alors plus une relation qu'une substance. C'est cette distinction qui lui permettra d'ailleurs de récuser l'opposition simple entre le réel et l'irréel : ce que nous dénommons « réel» s'oppose pour Goffman tout autant au jeu, au rituel, à l'apprentissage, à l'imposture, à l'expérimentation... qu'à l'« irréel » tout court. Bien entendu, toutes ces pratiques (jeu, expérimentation...) ont une existence effective, mais les acteurs ne

26 Kenneth G. Gergen, Le constructionisme social, Lonay, Delachaux et Niestlé, 2001.

27 Claude Lévi-Strauss, L'identité, Paris, PUF, 1983 ; Jean-François Bayart, L'illusion identitaire, Paris, Fayard, 1996.

${ }^{28}$ Luc Boltanski, Les cadres, Paris, Minuit, 1982 ; Jürgen Kocka, Les employés en Allemagne, 18501980, Paris, Éditions de l'EHESS, 1989.

29 Antonio Gramsci, Les cahiers de la prison (cahiers 6-9), Paris, Gallimard, 1983 ; Ernesto Laclau et Chantal Mouffe, Hegemony and Socialist Strategy, London, Thetford Press, 1985.

30 Judith Butler, Gender trouble, New York, Routledge, 1999 [1990]; Monique Wittig, La pensée straight, Paris, Balland, 2001.

${ }^{31}$ Jeffrey Alexander, The meanings of social life, Oxford, Oxford University Press, 2003 ; John Gagnon, Les scripts de la sexualité, Paris, Payot, 2008 ; Christian Salmon, Storytelling, Paris, La Découverte, 2007.

32 Erving Goffman, Les cadres de l'expérience, Paris, Minuit, 1991 [1974]. 
les considèrent pas comme ayant le même statut de réalité que d'autres actions. Plus que jamais la réalité est une affaire de cadrages et de juxtaposition des cadrages : à côté d'une expérience " directe " du monde, il existe toute une série d'expériences plurielles de la réalité à la suite de toute une série de transformations de cadrages. Avec subtilité, la problématique, toujours au cœur du paradigme de la représentation, change quelque peu de nature. Il ne s'agit plus seulement d'affirmer que la réalité dépend des représentations, mais de signaler que les "représentations » (les cadrages) ont la capacité d'accorder des différentiels de « réalité » aux pratiques sociales33.

Enfin, sans exhaustivité, une mention particulière des travaux sur la cosmopolitique s'impose dans ce contexte. Si bien des liens sont à établir avec l'ontologie de la production, notamment avec certains travaux autour de l'acteur-réseau, le concept de cosmopolitique est mobilisé par bien des anthropologues (ou philosophes) dans le but de redéfinir la relation entre la culture et la nature (des «métaphysiques cannibales » à Gaïa34) et, à terme, les entités qui peuplent le monde. La guerre des représentations atteint ici son paroxysme puisqu'il est plus que jamais question de la réalité de la réalité et de l'incommensurabilité radicale des regards. Ces versions de l'ontologie de la représentation, en mettant en question l'épistémologie moderne et l'épuration historique du monde qu'elle a effectuée (autour d'une connaissance réputée capable de trancher définitivement les controverses), imposent l'idée de l'inévitabilité des « multi-vers » ontologiques.

Retenons l'essentiel : la problématisation de la réalité spécifique à cette première famille de démarches socio-ontologiques s'organise autour du «langage ». Malgré les dénégations, et en dépit même de l'intérêt porté aux pratiques, au fond, tôt ou tard, la vie sociale finit souvent par être construite en analogie plus ou moins étroite avec le langage, devenant ainsi un espace étrangement trop ouvert ou trop fermé des contraintes. Trop ouvert : la vie sociale est abusivement associée à une série illimitée d'énonciations. Trop fermé : dans sa variante structurale notamment, elle est censée être limitée par des bornes symboliques indépassables 35 ou en tout cas par des cadres

33 L'analogie est forte avec ce que Danto caractérise comme la «transfiguration du banal» en tant que trait majeur de l'art contemporain : la capacité de l'artiste, de par sa légitimité sociale, à décréter le statut d'art - ou non - d'un objet. Cf. Arthur Danto, La transfiguration du banal, Paris, Seuil, 1989.

34 Eduardo Viveiros de Castro, Métaphysiques cannibales, Paris, PUF, 2009 ; Philippe Descola, Pardelà nature et culture, Paris, Gallimard, 2005 ; Isabelle Stengers, Cosmopolitiques, I et II, Paris, La Découverte, 2003 ; Bruno Latour, « Quel cosmos, quelles cosmopolitiques ? », dans Écologie politique, dir. Émilie Hache, Paris, Editions Amsterdam, 2004.

35 D'ailleurs, dans le structuralisme, cette plasticité ou cette rigidité ont été fortement accentuées par le passage d'une sémantique référentielle à une sémantique de relations internes. N'ayant plus de «matière » externe avec laquelle entrer en consonance, les différents systèmes de signes sont devenus des univers clos sur eux-mêmes et hautement contraignants. Pourtant, étrangement, c'est Lévi-Strauss lui-même qui a introduit la formule du «bricolage» pour faire référence aux multiples processus par lesquels les individus parviennent à l'agencement inédit de produits précontraints au cœur d'un nouvel espace de significations (Claude Lévi-Strauss, La pensée sauvage, Paris, Plon, 1962). Cette capacité combinatoire, sans être absolue, se prête cependant à un nombre tel de variations qu'il s'avère impru- 
institutionnels très rigides. Or, la vie sociale résiste à une assimilation analogique avec le langage à cause, justement, de la «nature » des contraintes dont elle est le théâtre. Il n'y a pas, du point de vue des coercitions, d'isomorphisme entre ces deux registres. La difficulté principale de ces démarches réside dans le risque d'aborder les résistances spécifiques du monde social exclusivement - ou essentiellement - à partir de considérations cognitives ou symboliques ${ }^{36}$.

Évitons tout malentendu : la dérive, dans certaines de ces socio-ontologies, vers la prise en compte des résistances de la réalité uniquement sous une forme symbolique est un risque effectif. Elle ne provient pas nécessairement de la négation d'une matérialité première, objective ou intransitive des faits sociaux (bien de ces démarches reconnaissent l'existence d'une réalité "extralinguistique »), mais du saisissement privilégié, voire exclusif, de la réalité à partir des catégories symboliques et de leur progressive évolution historique. Les formules sont parfois ambiguës. Puisque ce n'est que lors de leur " articulation » symbolique que les faits se constituent en tant que tels, c'est bien elle qui constitue l' « existence » d'un fait social et non sa référence «matérielle». Les résistances de la réalité ne sont cernées qu'en tant qu'incompatibilités symboliques.

Pourtant, ce n'est pas la seule manière de rendre compte des résistances de la réalité. Toujours au sein des socio-ontologies de la représentation, la résistance a aussi été mise à l'actif des "forces» sociales, une problématisation bien visible, par exemple, dans la manière dont Williams, à la suite de Gramsci, a mobilisé la notion d'hégémonie37. À la différence de la notion d'idéologie qui fait référence à un système formel d'idées fortement articulées entre elles, la notion d'hégémonie ne désigne pas une structure symbolique aussi homogène. À côté d'éléments faisant activement partie de l'hégémonie dominante, figurent toujours des éléments résiduels, formés dans une phase historique précédente, mais toujours présents dans une société donnée. Ce sont ces éléments « résiduels» ou " contre-hégémoniques » qui permettent justement d'exprimer des valeurs et des significations ne trouvant pas de place dans la culture dominante, et qui sont alors vécues et éprouvées à l'aide de ces formes rémanentes. Autrement dit, afin de rendre compte des incompatibilités symboliques entre éléments, l'analyse "sort» de la sphère symbolique proprement dite afin de tenir compte du travail des acteurs. Sournoisement, la socio-ontologie de la représentation s'ouvre en direction d'une autre forme de socio-ontologie.

Or, lorsque cette « ouverture » n'est pas explorée, comment nier que, tout en se défendant de toute dérive idéaliste, bien de ces perspectives risquent de réduire la réalité sociale, et ses résistances, sinon à leur seule perception, au moins à un pur

dent d'établir des limites ou des barrières définitives, en dépit de supposées incompatibilités symboliques.

36 Pierre Bourdieu, Le sens pratique, Paris, Minuit, 1980.

37 Raymond Williams, « Hegemony » (p. 108-114) et «Dominant, residual, and emergent» (p. 121127), dans Marxism and Literature, Oxford, Oxford University Press, 1977. 
travail de traduction culturelle ou institutionnelle ? La dérive vers un idéalisme sémiologique est particulièrement visible dans certains regards postmodernes décrivant les sociétés contemporaines à travers une déliquescence ontologique généralisée de la réalité, une prolifération de "distorsions » cognitives, un "flottement » généralisé des signes, l'existence de purs " simulacres », ou encore comme le lieu d'une réalité purement « virtuelle» ou même comme une société « liquide ». Or, énoncées de cette manière, ces visions n'ont que peu d'intérêt sociologique tant il est évident que les individus n'ont pas perdu, dans l'ordinaire de leur existence, le sens de la différence entre la « réalité » et la « fiction » ou le « virtuel». Rien d'étonnant alors si, face aux risques de ces excès, certains travaux proposent un travail de thérapie analytique afin de distinguer les usages acceptables ou non de la formule la « construction sociale de la réalités 8 ».

La force de cette première socio-ontologie est d'aborder les résistances de la réalité par la dialectique entre des faits «bruts»-objectifs et indépendants des individus - et des faits sociaux, au sens précis du terme, insérés donc dans la trame significative du monde social. La réalité est ce qui résiste, et le cœur de cette résistance (frontières cognitives, luttes d'intérêts...) s'exprime lors du travail de mise en représentation institutionnalisée du monde. Autrement dit, les faits sociaux n'existent qu'au travers d'un processus d'institutionnalisation, même si, une fois ce processus effectué, ils sont animés par des régularités objectives (comme c'est le cas, par exemple, de la monnaie ${ }^{39}$ ). En tout cas, avec la reconnaissance de cette dernière dimension, on est à la lisière d'un autre regard socio-ontologique. Comme on le verra ci-dessous, le grand intérêt de l'œuvre de Castoriadis est d'avoir formulé une version socioontologique totale de la représentation autour de l'imaginaire. Mais avant d'aborder sa proposition et ses difficultés, explicitons les contours théoriques des deux autres grandes démarches.

\section{Réalité et production}

Dans cette deuxième problématisation, ce qui retient vraiment l'attention ce sont les manières concrètes - «matérielles » et «pratiques »- de production de la vie sociale. Cette préoccupation de base explique d'ailleurs, dans ces socio-ontologies, la longue centralité de la dynamique entre l'objectivation (le devenir-monde de la vie sociale par sa fabrication) et l'aliénation (la perte de conscience que la réalité sociale est le fruit - et seulement le fruit - d'une fabrication).

${ }^{8}$ Ian Hacking, Entre science et réalité : la construction sociale de quoi ?, Paris, La Découverte, 2001. 39 Searle définit les faits sociaux comme subjectifs d'un point de vue ontologique (ils n'existent que parce qu'ils sont de nature sociale), mais objectifs d'un point de vue épistémologique (en dépit de leur genèse, ils peuvent être objet d'une connaissance visant à un établir une vérité objective). $C f$. John Searle, La construction de la réalité sociale, Paris, Gallimard, 1998 [1995]. 
Si la première grande formulation intellectuelle de cette vision se trouve dans le verum factum de Vico - «le critère et la règle de la vérité se trouvent dans l'avoir fait $40 »-$, ce n'est qu'avec le marxisme qu'elle va vraiment prendre corps. Ce qu'on a dénommé le paradigme de l'herméneutique du travail résume bien cette posture : la compréhension de la réalité exige un retour permanent vers son processus de production. Pour Marx, la conception de la réalité en tant que praxis suppose la capacité d'une réappropriation globale de la vie sociale en tant que pratique. Et à son tour cette compréhension ne peut qu'être pratique puisque « toute la vie sociale est essentiellement pratique ${ }^{41} »$. Feuerbach avait montré que derrière les dieux et à leur origine, il y avait l'aliénation des hommes. Marx s'est efforcé de montrer que toute la société, malgré ses opacités, n'est que pratique humaine. Tout un versant de l'œuvre de Marx tourne ainsi autour de la volonté de percer les différents projets d'occultation du monde en tant que pratique au travers des notions d'aliénation, d'idéologie, du fétichisme de la marchandise. Il s'agit de dévoiler la vérité derrière l'illusion, de séparer la conscience de la fausse conscience. La réalité est travail, et cette nouvelle conception de la réalité exige un nouveau critère de vérité : c'est « dans la pratique que l'homme a à faire la preuve de la vérité, c'est-à-dire de la réalité et de la puissance de sa pensée, la preuve qu'elle est de ce monde 42 ». Rien d'étonnant que certains aient pu établir un lien entre le marxisme et le pragmatisme43.

La praxis apparaît ainsi comme un concept central de la socio-ontologie marxiste : c'est par le travail que les hommes parviennent - ou pourraient parvenir - à se cerner « comme les acteurs et les auteurs de leur propre histoire 44 ». L'analyse que Marx fournit du travail dans le capitalisme est en filiation étroite de cette problématique : c'est le travail qui produit de la valeur, ou dans des mots plus précis, seul le travail a une véritable dimension créatrice. Tous les objets créés par l'homme - comme toutes les institutions d'ailleurs - ne sont que du travail cristallisé, toutes les marchandises sont $\mathrm{du}$ «travail mort». L'essentiel de la phénoménologie économique de Marx tourne ainsi autour de la volonté de dé-réifier les catégories économiques en montrant jusqu'à quel point elles ne sont rien d'autre que des mystifications du travail humain. C'est même par la praxis, et l'objectivation essentielle dont elle est porteuse, que l'homme se fait lui-même, qu'il devient ce qu'il fait. Certes, pour Marx il y a

40 Giambattista Vico, Principes d'une science nouvelle relative à la nature commune des nations, Paris, Éditions Nagel, 1953 [1744]. Cependant, écrite en 1744, la Scienza nuova enferme deux limitations essentielles. La première est liée au rôle que Vico accorde encore à Dieu : si les hommes font l'histoire, ils ne le font qu'avec le concours de la Providence. La seconde, encore plus importante, est qu'au-delà de la formule, Vico n'a que très superficiellement évoqué les raisons et les processus par lesquels les hommes font effectivement l'histoire.

$4^{1}$ Karl Marx, "Thèses sur Feuerbach » [1845], dans Karl Marx et Friedrich Engels, L'idéologie allemande, Paris, Éditions Sociales, 1976 [1844], p. 3.

42 Ibid., p. 1.

43 Richard J. Bernstein, Praxis and action, Pennsylvania, University of Pennsylvania Press, 1971.

44 Karl Marx, Misère de la philosophie [1847], dans Euvres, Économie, t. 1, Paris, Gallimard, 1965, p. 84 . 
toujours, précédant l'homme, un « substrat matériel », une « nature », une "matière première » toujours déjà-là, mais l'essentiel de son effort est de se pencher sur le travail et l'univers des objets créés et recréés par le travail, c'est-à-dire, cet échange inépuisable entre l'homme et la nature au sein de configurations sociales de plus en plus autonomes et épaisses. Autant dire que la notion de praxis a trois significations chez Marx : ontologique, anthropologique et historique. L'influence durable de ce cadre d'interprétation sur Castoriadis est évidente.

Aucun autre texte de la tradition marxiste ne montre mieux cette attitude socioontologique que l'analyse que Georg Lukács a consacrée au fétichisme de la marchandise 45. La notion fait explicitement référence à la transformation de processus vifs en choses mortes, à la mutation des êtres en choses, qui ne tardent pas à se présenter à l'homme comme une "deuxième nature " aliénée. Toutes les formes d'objectivité et toutes les formes correspondantes de subjectivité sont marquées par la structure de la relation marchande, ce qui produit autant une pseudo-objectivité qu'une pseudo-subjectivité. Dans le capitalisme, l'homme devient le spectateur impuissant d'un ensemble réifié de processus qu'il découvre comme étranger et menaçant.

Bien entendu, la réduction de la réalité au seul travail n'est pas la seule voie possible de cette matrice socio-ontologique. De Wright Mills à Habermas, sans oublier les premiers travaux de Baudrillard et même l'étude sur la dialectique de Sartre, il s'agira, souvent dans le sillage critique du marxisme, de souligner à la fois la force de cette conception productive de la réalité, tout en la dissociant de son assise exclusive autour de l'herméneutique du travail. Un projet encore plus explicite dans les démarches qui, comme c'est le cas avec le réalisme critique, ont pris une distance plus grande et plus marquée avec le marxisme46. Pour Anthony Giddens, par exemple, la dualité du structurel souligne la récursivité permanente de la vie sociale, et permet d'avoir une vision ontologiquement dynamique de la production incessante de la réalité : les structures n'existent qu'au niveau de l'action et les actions ne peuvent exister que grâce aux structures. La réalité est cernée comme le fruit de l'activité humaine - mais bien au-delà de la seule herméneutique du travail47. D'autres, comme Bhaskar, préfèrent souligner davantage la transformation que la production : dans le "modèle transformationnel de l'activité sociale", les acteurs ne sont pas censés fabriquer la société, mais se limitent, à partir de formes sociales toujours déjà-là, à la reproduire et à la transformer ${ }^{48}$. Toujours dans une intuition proche, Archer va

\footnotetext{
45 Georg Lukács, Histoire et conscience de classe, Paris, Minuit, 1960 [1923], p. 109-256.

46 Margaret Archer et al., Critical realism : essential readings, London, Routledge, 1998.

47 Anthony Giddens, La constitution de la société, op. cit.

48 Roy Bhaskar, The possibility of naturalism, Harvester Wheatsheaf, Hemel Hempstead, 1989. Le réalisme critique de Bhaskar propose une vision multi-stratifiée de la réalité, en différenciant entre un niveau profond, "réel », non directement observable, qu'il distingue de deux autres niveaux, l'« empirique » et l'« actuel». Le but est de faire reconnaître que les structures (la « société »), tout en
} 
distinguer, grâce au primat qu'elle accorde à la dimension temporelle (toute production vient toujours " après » une réalité initiale), entre une morphogenèse (transformation) ou une morphostase (reproduction) de la réalité - mais dans les deux cas, l'articulation entre l'action et la structure passe par un décalage temporel : le passé, sous la forme de structures, précède les actions dans le présent, et à leur tour, les actions peuvent modifier les structures dans le futur par leur déroulement49. Dans ces visions, l'articulation plus ou moins univoque entre l'acteur et le système (par la socialisation) cède le pas à des problématiques plus ouvertes, voire plus contingentes, de production et de reproduction de la vie sociale. La réalité sociale est indissociablement contraignante et habilitante.

Si l'agency a élargi la socio-ontologie de la production au-delà du seul travail, la production de la vie sociale est cependant restée cantonnée aux seuls facteurs sociaux et humains. C'est cette homogénéité ontologique qui est mise en question depuis quelques décennies. La production de la réalité est abordée à partir de l'assemblage d'éléments autrement plus hétérogènes. La notion de réseau, dans toute sa polysémie, résume mieux que n'importe quel autre concept cette perspective, sans doute parce qu'elle s'insère dans la conception d'un connexionnisme universel5o. Les visions ontologiques les plus conséquentes défendent l'idée d'une « relationnalité » radicale : les associations ne s'établissent pas vraiment entre éléments déjà constitués, donc préalables et indépendants à la relation, mais ce sont les relations qui, en mettant en relation des «ingrédients» divers, les constituent véritablement en tant qu'éléments ${ }^{51}$. La caractéristique de chaque élément n'est plus considérée comme un trait intrinsèque ; il dépend du réseau - ou du système chez Luhmann - dans lequel il s'insère. L'association et l'assemblage deviennent le cœur d'une autre version de la socio-ontologie de la production. L'attention se centre sur les dispositifs d'objectivation et le travail d'assemblage : la stabilité des objets et des pratiques n'est conçue que comme le résultat complexe et temporaire d'assemblages matériels.

Une mention spéciale doit être faite ici à l'œuvre de Latour où se trouve une des versions les plus radicales de cette perspective ontologique. Sa tentative consiste à dépasser la dichotomie entre la société et l'individu en récupérant les objets sur lesquels repose la vie sociale52. Toutes les interactions sont encadrées par une myriade

préexistant aux individus, dans le sens où c'est quelque chose qu'ils n'ont jamais directement fait, n'existent qu'en vertu de leur activité.

49 Margaret Archer, Realist social theory, Cambridge, Cambridge University Press, 1995.

50 Luc Boltanski et Ėve Chiapello, Le nouvel esprit du capitalisme, Paris, Gallimard, 1999, p. 208-230.

51 John Law et John Hassard (dir.), Actor network theory and after, London, Blackwell, 1999 ; Manuel Castells, L'ère de l'information, t. 1, La société en réseaux, Paris, Fayard, 1998 [1996]; Fernando J. García Selgas, Sobre la fluidez social. Elementos para una cartografía, Madrid, CIS, 2007 ; Manuel DeLanda, A new philosophy of society, New York, Continuum, 2006 ; Harrison C. Whyte, Identité et contrôle, Paris, Éditions de l'EHESS, 2011 [2008]. Sur ce point, l'enchevêtrement entre le constructivisme radical et la socio-ontologie de la production est évident.

52 Bruno Latour, Changer de société. Refaire de la sociologie, Paris, La Découverte, 2006. 
d'«actants » dispersés dans l'espace et dans le temps qui, dans leurs enchevêtrements pluriels, ne constituent nullement une structure, mais permettent cependant d'expliquer les effets de structuration à l'œuvre, grâce à une série de connexions matérielles. Pour Latour, cette inflexion exige de ne plus considérer les objets comme purs intermédiaires des humains, mais au contraire, et dans un renversement saisissant de la célèbre formule de Durkheim, de traiter les choses comme des faits sociaux. Le noyau dur de cette perspective est donc de critiquer la réduction de l'association (en fait de la fabrication de la vie sociale) au seul modèle de l'interaction sociale (et du travail). L'ontologie n'est plus uniquement sociale, puisqu'elle passe par la constitution d'une série de réseaux permettant de stabiliser diverses objectivations grâce à différentes alliances entre acteurs humains et nonhumains53. Plus que jamais, l'ontologie de la vie sociale est cernée à partir de sa fabrication et ce qui prime est bien l'agencement entre éléments (l'ontological glue entre eux). Pourtant, et en dépit d'une indéniable nouveauté, nous sommes toujours dans la socio-ontologie de la production, même si l'ancienne figure du «travailleur » a été remplacée par un souci d' « ingénieur».

Malgré son importance, le travail de Latour doit être mis en relation avec d'autres travaux qui explorent aussi, à la lumière d'une ontologie élargie de la production, les processus de fabrication des « objets» scientifiques (ADN, microbes...) ou avec les débats sur l'avènement d'une nouvelle ère géologique autour de l'anthropocène. L'idée des sciences en tant que productrices des mondes qui « meublent» l'univers de nouveaux objets, s'impose progressivement54, et avec elle l'idée d'une ontologie de la production d'un nouveau genre - Ian Hacking n'a pas hésité à parler, afin de caractériser ces capacités tous azimuts de création et de fabrication, d'« ontologies historiques55 ».

Ceci nous ramène à notre préoccupation centrale - la spécificité de cette voie socio-ontologique et la manière dont elle envisage les résistances de la réalité. Si les résistances proprement "matérielles » de la réalité ne sont pas complètement étrangères à ces perspectives, ce n'est nullement cet aspect qui est vraiment retenu; les résistances sont surtout abordées par la tension entre l'objectivation consciente de l'homme et le constat d'aliénation - sous la forme d'un « oubli» de cette vérité ou d'une «impuissance» face à la production en elle-même. C'est dans ce sens d'ailleurs que cette socio-ontologie a souvent repris - et prolongé - la notion d'aliénation de Marx - un aspect patent aussi dans le travail de Castoriadis. La résistance de la réalité se colore alors souvent d'un aspect "pathologique ": le sujet s'avère incapable de se reconnaître dans ce qu'il fait. Certes, dans ces interprétations, l'aliénation peut être soit une dimension ontologique inévitable de la praxis humaine,

53 Bruno Latour, Nous n'avons jamais été modernes, Paris, La Découverte, 1991.

54 Sophie Houdart et Olivier Thiery (dir.), Humains, non-humains. Comment repeupler les sciences sociales, Paris, La Découverte, 2011.

55 Ian Hacking, Historical Ontology, Cambridge, Harvard University Press, 2002. 
soit, au contraire, le résultat surmontable d'une dimension historique. Mais, dans tous les cas, elle est au cœur de la définition de la réalité comme ce qui résiste. Même chez quelqu'un comme Latour, en apparence très éloigné de cette filiation critique, cette dialectique est à l'œuvre. Qu'il s'agisse de quasars, du PIB ou des microbes, ce sont les dispositifs, permettant - ou non - l'inscription et la stabilisation dans le réel de chacun d'entre eux, qui sont au centre de l'analyse. Double résultat : d'un côté, le monde entier devient un immense laboratoire (où « tout » peut être assemblé) et de l'autre côté, la réalité est ce qui résiste de par la force temporaire de ce qui a été assemblé (la pérennité des « êtres » s'explique par la force des alliés et des porte-parole des réseaux assemblés). Étrangement, le passage par l'«interobjectivité » du monde ne fait que porter à son paroxysme le présupposé subjectiviste d'un univers susceptible de fabrication continue et son corollaire d'aliénation.

Si les points de contact entre cette démarche et la socio-ontologie de la représentation (notamment avec les démarches constructivistes) sont notoires, elles n'en sont pas moins porteuses de deux problématisations différentes. Ici, la résistance de la réalité, malgré le volontarisme souvent de mise dans ces travaux, est bien mieux cernée dans ses aspects proprement matériels. Certes, la conscience du caractère produit de la réalité sociale finit parfois par faire chair avec l'idée - quelque peu différente d'une réalité pouvant être, sans répit, transformée par la volonté humaine. Et pourtant, et parfois à leur corps défendant, ces démarches ne cessent d'ouvrir inlassablement la question des résistances du monde social : une fois objectivés, les faits sociaux ne sont pas modifiables, ou difficilement, par la volonté humaine. On verra cidessous l'importance de cette dialectique - et de cette tension - dans la socioontologie de Castoriadis.

\section{Réalité et action}

Tout en s'accordant sur le postulat que la réalité est ce qui résiste, la socio-ontologie de l'action propose une troisième problématisation. La spécificité de l'action est d'attirer l'attention sur les moments effectifs des problématisations de la conduite par les résistances de la réalité. Comme on le verra, et c'est là que réside la véritable spécificité de cette démarche, la problématisation du rapport à la réalité à partir de l'action impose presque d'emblée à la fois l'évidence d'un monde social supposé contraindre fortement les conduites et les constats du caractère souvent hautement problématique des contraintes sur les acteurs. Pour comprendre cette tension, il est nécessaire de différencier entre, d'une part, des coercitions pouvant opérer indépendamment de leur reconnaissance par les acteurs et, d'autre part, la reconnaissance explicite et "vécue » des moments de friction avec la réalité. Rien de surprenant à cet égard : la prise en compte des résistances de la réalité est ce qui sépare justement l'action de la fiction, les rêveries ou le sommeil. Or, autour de cet accord, deux grandes positions se différencient selon que le caractère problématique des résistances de la réalité soit placé en amont ou en aval de l'action. 
Aucune autre démarche n'a mieux abordé cette question que le pragmatisme et sa volonté de faire de la réussite ou de l'échec d'une action son critère central d'évaluation ${ }^{6}$. Le grand partage s'établit entre des actions qui, suivant des règles ou des croyances, s'insèrent sans problème dans le monde, et des actions qui, au contraire, rencontrent des situations problématiques et, de ce fait, obligent à inventer de nouvelles règles 57 . Autant dire que les résistances de la réalité se constatent en aval de l'action. Dans ce sens, il faut reconnaître toute la pertinence de la relecture que Joas a faite de la tradition pragmatique (et la distance de cette démarche avec la vision de Castoriadis) : toute action est créatrice parce que, dans son devenir même, elle se doit de résoudre et de dénouer des obstacles 58 . Cependant, dans cette démarche, les clés de la réussite ou de l'échec d'une action ne sont jamais immédiatement données par un état matériel du monde; les raisons et les facteurs de réussite et d'échec se construisent dans le cadre des relations sociales. Un des grands mérites du pragmatisme est justement d'avoir souligné à quel point l'acteur est dépendant, lors de l'évaluation de la réussite ou de l'échec de son action, du contexte interindividuel et collectif dans lequel il agit59. En prenant cette position, les pragmatistes ne récusent pas l'existence d'une réalité extérieure aux acteurs, mais ils soulignent que l'action de l'environnement est indissociable des significations sociales. Surtout, comme pour la socio-ontologie de la praxis, la pratique est le principe de vérification - voire de vérité - de la relation entre l'action et la réalité6o. C'est dans la pratique que la pensée doit donc faire la preuve de sa pertinence; les pragmatistes pensent même les théories comme des outils grâce auxquels l'esprit opère dans la réalité.

Or, le succès d'une action, ici et maintenant, ne présage en rien de sa fortune future, puisque les individus créent constamment de nouveaux langages, et qu'en plus, il existe différentes manières (scientifique, morale, esthétique...) de se rapporter à la réalité61. Ce qui questionne en profondeur l'idée d'une sanction-couperet objective et

\footnotetext{
56 William James, Le pragmatisme, Paris, Flammarion, 2007 [1907]. Reconnaissons cependant que ce n'est pas toujours l'action et son rapport à la réalité qui est au cœur du pragmatisme, mais plutôt la question de la stabilisation du monde entre les acteurs - le monde qui «tient » les individus. Le pragmatisme n'est pas toujours une philosophie de l'action; il est bien davantage une philosophie de la pratique et de la réalité en train de se faire. Dans sa version de sociologie pragmatique, Boltanski et Latour ont fortement accentué cette dimension. Autrement dit, la réalité est tout autant sinon davantage étudiée à partir de la socio-ontologie de la production que de l'action à proprement parler.

57 Jean-Pierre Cometti, Qu'est-ce que le pragmatisme?, Paris, Gallimard, 2010, p. 339-340.

$5^{8}$ Hans Joas, La créativité de l'agir, Paris, Cerf, 1999 [1992].

59 Notons-le, ce souci est constant à bien d'autres démarches, chaque fois qu'il est question des moments de problématisation des significations, lorsque les routines ou les schémas de typifications, et plus largement le sens commun, s'avèrent insuffisants pour réintégrer et absorber des "sauts» ou des « ruptures » dans les habitudes quotidiennes. Cf. Harold Garfinkel, Recherches en ethnométhodologie, Paris, PUF, 2007 [1967].

60 William James, Le pragmatisme, op. cit., p. 113 : «La méthode pragmatique vise à interpréter chaque notion en fonction de ses conséquences pratiques ».

${ }^{61} \mathrm{C}$ 'est pourquoi, comme le souligne Putnam, la correspondance avec la réalité est, à la lettre, une formule creuse pour définir la vérité ; encore faut-il être capable de préciser les critères de confirmation.
} 
immuable de la réalité comme principe de vérité. Si la différence du pragmatisme avec un réalisme naïf (et l'idée que la réussite ou l'échec d'une action sont le résultat direct et immédiat des coercitions du monde) est patente, il n'en reste pas moins que le processus lui-même de sanction par la réalité est souvent sous-analysé. En fait, il y a une ambiguïté permanente dans le pragmatisme. Pour Dewey, par exemple, tout jugement résulte d'une situation problématique, l'important est donc de « découvrir ce que sont le ou les problèmes qu'une situation problématique pose à l'enquête 62 "». Cependant, il n'est jamais entièrement clair sur ce qui prime au moment de la définition de la situation problématique - une expérience d'entrave pratique ou un désaccord entre acteurs ${ }^{63}$. D'ailleurs, dans les aspects les plus pragmatiques de son travail, comme dans ses études sur la science moderne et les pratiques d'expérimentation, il est possible de repérer la même hésitation chez Latour64. Comme Dewey, il ne tranche jamais vraiment entre ces deux perspectives, tant ses études oscillent entre, d'une part, une vision accordant une place plus ou moins effective et directe aux objets, en fait à la matérialité intrinsèque du monde dans les jugements; et, d'autre part, une vision plus nietzschéenne où c'est l'épreuve de force qui semble - parfois en dehors de toute contrainte matérielle - trancher une controverse. Bien entendu, les deux processus peuvent être complémentaires entre eux, mais ils n'en définissent pas moins deux questions différentes.

C'est autour de ce point que s'organise une seconde variante de la socio-ontologie de l'action. Elle problématise autrement le moment de la problématisation de l'action par la réalité en reconnaissant le fait que, d'ordinaire, le couperet pratique des actions dans la vie sociale repose davantage sur un postulat imaginaire que sur des sanctions concrètes 65 . Autant dire que la certitude des résistances de la réalité s'établit en amont de l'action. C'est donc en prenant acte de la complexité des problématisations en aval de l'action par la réalité que ces démarches élargissent la focale 66 .

Cf. Hilary Putnam, Pragmatism : an open question, Oxford, UK \& Cambridge, USA, Blackwell, 1995 [1992].

62 John Dewey, Logique : la théorie de l'enquête, Paris, PUF, 1993 [1938], p. 173.

63 John Dewey, La formation des valeurs, Paris, La Découverte, 2011.

64 Bruno Latour, Aramis, ou l'amour des techniques, Paris, La Découverte, 1992 et Les microbes, guerre et paix, Paris, La Découverte, 2001 [1984].

65 Prendre pleinement acte de cette spécificité de la vie sociale, amène à établir quelques distances face aux versions canoniques de la tradition pragmatique. Dans la vision pragmatiste, l'enquête ne se produit que lorsqu'un acteur, individuel ou collectif, se heurte à une difficulté qui engendre un doute ou une incertitude. La manière dont Erving Goffman (op. cit.) étudie la rupture de cadrages ou la façon dont la notion d'épreuves a été mobilisée dans la sociologie de l'acteur-réseau ou de la justification, partage cette intuition.

66 D'ailleurs, le destin d'une action ne peut pas ne pas se complexifier dès qu'on prend acte que toute action - ou presque - en fonction de la temporalité d'évaluation choisie est susceptible, au moins potentiellement, de voir sa signification initiale se renverser. Ce processus, parfois dénommé conséquences non intentionnelles de l'action, est à l'œuvre dans des représentations aussi contrastées que la théodicée 
En effet, bien des actions, y compris lorsqu'elles vont à l'encontre de certaines règles ou attentes collectives, ne connaissent pas vraiment, à cause de la particulière consistance de la vie sociale, de «coups d'arrêt ${ }^{67}$ ». L'idée d'un environnement social et culturel à détente durable et constante, établissant des limites indépassables, et permettant de dégager des accords pragmatiques stables et solides sur ce qu'est la réalité (grâce au succès ou à l'échec des actions), n'est qu'un cas de figure parmi bien d'autres - et loin d'être le plus fréquent. Hormis quelques situations, là où l'échec d'une action, après évaluation et élaboration explicites, prouve pratiquement à l'acteur, comme aux autres, son erreur, l'essentiel de la vie sociale se déroule à l'abri de ce type de considérations. Les critères d'évaluation de l'action seraient donc bien plus labiles que ce que laisse souvent sous-entendre le sens commun ; dans bien des situations, l'action se déroule dans une sorte de clair-obscur que l'acteur ne s'efforce pas, ou rarement, d'éclaircir'88.

Ces travaux mettent ainsi en question l'idée que les acteurs s'engagent, lorsqu'ils rencontrent une résistance ou un désaccord lors de leur action, dans un processus de clarification conceptuelle69. À part quelques situations de ce type, où de toute évidence l'intérêt pratique cède le pas à une posture intellectuelle ou de justification, les acteurs continueraient quotidiennement à agir en dépit de divers démentis ou tensions, y compris interactifs, que la réalité leur oppose. Les individus peuvent longtemps rester immuns, voire indifférents, aux réactions plus ou moins hostiles de leur environnement s'ils disposent de protections pratiques suffisantes ${ }^{70}$. Ce qui entraîne deux conséquences. D'une part, cela oblige à prendre acte de l'importance du temps dans le saisissement des heurts avec la réalité - aspect qui ne retient pas toujours l'attention qu'elle mérite ${ }^{11}$. D'autre part, cela invite à ne pas aborder cette problématique uniquement comme une question se décidant en aval, et pouvant alors prendre,

chrétienne, la ruse de la Raison hégélienne, les fonctions latentes, l'aliénation ou encore les effets pervers.

67 Danilo Martuccelli, La consistance du social, Rennes, PUR, 2005.

68 C'est par exemple ce qui ressort des enquêtes sur l'évaluation du travail scolaire ( $c f$. Anne Barrère, Les lycéens au travail, Paris, PUF, 1997) ou sur le fonctionnement des services psychiatriques où, une fois une hospitalisation effectuée, il est difficile de distinguer entre des comportements sains ou pathologiques ( $c f$. David L. Rosenhan, "Être sain dans un environnement malade », dans L'invention de la réalité, dir. Paul Watzlawick, Paris, Seuil, 1988).

69 Erhard Friedberg, Le pouvoir et la règle, Paris, Seuil, 1993.

$70 \mathrm{C}$ 'est par exemple la conclusion à laquelle parvient Declerck à propos de la trajectoire de certains exclus - pas tous : leur indifférence à l'environnement, rendu possible par leur entourage familial, peut les «protéger» pendant longtemps d'une déchéance personnelle. Cf. Patrick Declerck, Les naufragés, Paris, Plon, 2001, p. 320.

${ }^{71}$ C'est, par exemple, le postulat implicite des ouvrages, par ailleurs très stimulants, de Morel sur les décisions absurdes : il ne souligne jamais assez les laps de temps longs s'écoulant avant qu'une action ne rencontre une opposition ou ne soit l'objet d'une problématisation correctrice. $C f$. Christian Morel, Les décisions absurdes, Paris, Gallimard, 2002 et Les décisions absurdes, II, Paris, Gallimard, 2012. 
comme dans le célèbre exemple de la dissonance cognitive, des proportions extrêmes $^{72}$, mais comme une certitude postulée en amont et d'emblée par les acteurs.

Le glissement de l'aval vers l'amont comme lieu de problématisation du rapport entre l'action et la réalité est bien visible dans la lecture que Schütz propose du Quichotte73. Comment Don Quichotte parvient-il à neutraliser l'existence d'une réalité qui va à l'encontre de ses croyances ? La proposition de Schütz est que, pour y parvenir, Don Quichotte est contraint de gérer les contradictions à l'aide d'interprétations ad hoc (comme celle, par exemple, produite par les enchanteurs) permettant la coexistence et la compatibilité entre différents sous-univers de signification. Pourtant, menée à partir de considérations exclusivement cognitives, l'analyse de Schütz sous-estime largement les soubassements proprement pratiques des aventures du Quichotte, à savoir que les arrangements pratiques avec l'environnement peuvent être multiples et se révéler actifs pendant des laps de temps plus ou moins longs. « N'importe quoi » n'est pas opératoire dans la vie sociale. Et pourtant, bien des attentes, voire des croyances (y compris parmi les plus «délirantes», si on se réfère toujours au Quichotte), ne sont en aucun cas immédiatement démenties comme le montre l'analyse proposée par Nabokov qui finit par distinguer vingt actions couronnées de "succès » et vingt autres sanctionnées par un « échec74 ». Certes, une partie de l'explication réside dans des mécanismes de défense proprement cognitifs. Mais cela n'est qu'une partie de la vérité. Si cela se révèle possible, c'est aussi parce que, très pratiquement, les acteurs peuvent souvent continuer à opérer dans la réalité sociale, avec une efficacité certaine, et pendant un laps de temps plus ou moins long, à l'aide de représentations pourtant jugées comme fausses ou discutables à partir d'une croyance dominante75. Autant dire que les résistances escomptées de la réalité sont davantage à cerner comme une question imaginaire (en amont donc de toute action et heurt concrets) que comme une affaire pragmatique et en aval.

Il s'agit d'un aspect bien reconnu, au moins implicitement, par Robert K. Merton à l'aide de la notion de prophétie autoréalisatrice. Comment est-ce possible qu'en s'appuyant sur des représentations « fausses » (disons controversées), un acteur puisse finir par produire des conséquences «vraies» (disons réelles76) ? D’emblée, Mer-

${ }^{72}$ Léon Festinger, Hank Riecken, Stanley Schachter, L'échec d'une prophétie, Paris, PUF, 1993 [1956]. 73 Alfred Schütz, « Don Quichotte et le problème de la réalité » [1953], dans Essais sur le monde ordinaire, Paris, Le Félin, 2007.

74 Vladimir Nabokov, Littérature III. Don Quichotte, Paris, Fayard, 1992 [1983].

75 Bien entendu, tout n'est pas arbitraire, mais l'éventail des possibilités est tel, qu'il a parfois été légitime de conclure que presque toutes les solutions peuvent s'adapter à presque tous les problèmes à condition qu'ils émergent à peu près en même temps. $C f$. James G. March et Johan Olsen, Rediscovering institutions, New York, The Free Press, 1989, chapitre IV.

${ }^{76}$ Le problème n'a pas échappé à Merton lui-même qui, pour y répondre, fait référence au temps comme critère ultime d'évaluation (par exemple à propos des conséquences de l'exclusion des travailleurs noirs des syndicats américains). Cf. Robert K. Merton, Éléments de théorie et de méthode sociologique, Paris, Plon, 1965 [1949]. 
ton a différencié entre une conception plutôt institutionnelle de la prophétie autoréalisatrice (elle n'opère que dans la mesure où elle devient une croyance socialement partagée) et une conception trop subjectiviste de ce qu'il avait lui-même baptisé à l'origine le "théorème de Thomas ", et qui risque de faire oublier à quel point l'action sociale est bornée par un grand nombre de facteurs collectifs77. Or, comme Merton en viendra à le reconnaître lui-même, la liberté de définition subjective des situations n'est pas illimitée, puisque «si les individus ne définissent pas des situations réelles comme réelles, celles-ci seront néanmoins réelles dans leurs conséquen$\operatorname{ces}^{78} »$. Or, en évoquant cette dimension, et sans qu'il en soit peut-être entièrement conscient, Merton "glisse » d'une analyse fondée - et uniquement fondée - sur des observations factuelles en aval (pour controversées qu'elles puissent être comme c'est le cas, y compris pour des raisons temporelles, dans les prophéties autoréalisatrices) vers une interprétation s'appuyant sur une croyance en amont - la certitude cognitive de l'existence des résistances effectives dans la réalité malgré la difficulté à l'établir. La problématisation de l'action par la réalité devient davantage une supposition qu'une expérience, la certitude en amont prime sur les constats en aval. Merton ne l'affirme pas explicitement, mais c'est bel et bien le principal postulat de cette seconde variante de l'onto-sociologie de l'action : toute conduite présuppose que la réalité est ce qui résiste, mais dans la mesure où la résistance peut s'avérer opaque au niveau de l'action elle-même, il faut reconnaître le rôle d'éléments proprement imaginaires dans l'établissement du caractère incontournable de la réalité.

Le propre de cette voie de la socio-ontologie de l'action tient justement à ce que les individus opèrent au sein d'un environnement où les limites sont fondamentalement indéterminées du point de vue de la pratique et systématiquement présentes au niveau de l'imaginaire. La relation entre l'action et la réalité, pour ouverte qu'elle s'éprouve dans les faits - en aval donc -, est inséparable du postulat de l'existence de moments inévitables de corrections - c'est-à-dire, en amont. Autant dire que ces corrections sont plus une croyance - un "principe de réalité »-qu'une expérience directe (si elles sont toujours supposées, elles sont bien plus rarement éprouvées); et en même temps, elles sont bel et bien à la base de la croyance fondatrice de l'expérience de l'action79.

La socio-ontologie de l'action oscille ainsi entre, d'une part, une conception pragmatique et proprement factuelle des résistances de la réalité et, d'autre part, une

\footnotetext{
77 C'est ainsi que, comme le montre «l'effet Pygmalion », le fait de considérer certains élèves comme particulièrement aptes à la connaissance induit chez les enseignants un traitement spécifique, qui favorise, dans certaines limites, un réel processus d'apprentissage. Pourtant, comme on le sait, l'exemple n'est pas toujours probant à la fois pour des raisons «objectives" et pour des raisons tenant à la noninstitutionnalisation collective des attentes enseignantes. $C f$. Robert A. Rosenthal et Lenore Jacobson, Pygmalion à l'école, Tournai, Casterman, 1983 [1968].

78 Robert K. Merton, «Social problems and sociological theory », dans Contemporary social problems, dir. Robert K. Merton et Robert Nisbet, $4^{\mathrm{e}}$ éd., New York, Harcourt Brace Jovanovich, 1976, p. 22.

79 Danilo Martuccelli, Les sociétés et l'impossible, Paris, Armand Colin, 2014.
} 
conception accentuant, souvent en amont de toute conduite effective, la force de la croyance dans les résistances immédiates de la réalité. Dans aucune autre démarche socio-ontologique, les résistances de la réalité ne semblent alors aussi évidentes ; et en même temps, paradoxalement, aucune autre variante socio-ontologique n'a autant de mal à les établir précisément. Au point que la socio-ontologie de l'action finit parfois par faire alliance avec la socio-ontologie de la représentation afin de cerner les résistances de la réalité.

\section{Castoriadis et le projet d'une socio-ontologie totale}

$\mathrm{Au}$ vu de ces théorisations, la socio-ontologie de Castoriadis possède un intérêt majeur parce qu'elle permet, bien mieux que d'autres, d'analyser en détail les possibles (ou difficiles) enchaînements entre les trois perspectives que nous venons de détailler. C'est en affirmant la réalité-comme-production, mais en constatant l'échec de toute planification achevée de la vie sociale (la bureaucratisation), qu'il a bâti une conception particulière de la praxis-comme-création qui a fini par ancrer son ontologie davantage du côté de la représentation - l'imaginaire radical - que du côté de l'action et de l'étude, sous cette modalité, des résistances de la réalité. Malgré son souhait de proposer une socio-ontologie totale, son œuvre s'organise autour d'une notion de création qui n'est finalement qu'une variante de la conception de la réalitécomme-représentation (l'imaginaire radical). Son œuvre permet ainsi de revenir sur la principale difficulté de bien des socio-ontologies - rendre compte du caractère effectif des résistances de la réalité.

En tenant en arrière-plan les perspectives socio-ontologiques présentées, explicitons les étapes analytiques de l'ontologie de la création chez Castoriadis. Le point de départ en est explicitement placé dans le sillage des travaux de Marx et d'une ontologie de la production. Les premières analyses que Castoriadis consacre à la bureaucratie, dès la fin des années 1940, ont pour objectif de mettre à jour la nouvelle structure d'exploitation présente dans les régimes soviétiques. Mais dès le départ sa réflexion vise à cerner, au niveau de la production économique, la contradiction principale $\mathrm{du}$ monde moderne. En Russie comme dans l'Occident capitaliste, Castoriadis décèle un même mécanisme d'exploitation. «La planification bureaucratique n'est rien d'autre que l'extension à l'ensemble de l'économie des méthodes créées et appliquées par le capitalisme dans la direction "rationnelle" des grandes unités de production 80 ». Autant le capitalisme de l'entreprise bureaucratisée que la planification de l'État omniprésent sont des manifestations d'une même forme sociale, le capitalisme bureaucratique. Or, et c'est ici que se trouve la véritable source de la pensée ultérieure de

\footnotetext{
80 Cornelius Castoriadis, «La révolution prolétarienne contre la bureaucratie » [1956], dans La société bureaucratique, t. II, La révolution contre la bureaucratie, Paris, Union Générale d'Éditions, 1973, p. 278.
} 
Castoriadis sur la création, le triomphe de la rationalisation dans la modernité ne signifie nullement la disparition des capacités alternatives d'action.

Face à la bureaucratisation capitaliste, Castoriadis oppose en effet, en se réclamant explicitement de la tradition du conseillisme ouvrier, la gestion ouvrière de la production $^{81}$. Pour Castoriadis, il existe un conflit irrépressible entre l'ouvrier individuel et les ouvriers auto-organisés informellement d'un côté, et le plan de production et d'organisation imposé par l'entreprise de l'autre côté. C'est là qu'il décèle la présence, toujours à l'œuvre, d'une organisation ouvrière informelle et fragmentaire, en opposition à l'organisation formelle de la production, mais sans laquelle il ne pourrait pas y avoir à proprement parler de production. L'absurdité essentielle du capitalisme (comme d'ailleurs de la planification) et les limites de sa supposée rationalité résident à ce niveau : il doit à la fois exclure les ouvriers de la direction de leur propre travail et, afin de ne pas s'écrouler, faire appel aux groupes informels d'ouvriers et à leur créativité afin de pouvoir assurer la production ${ }^{82}$. Plus tard, Castoriadis dira que ce n'est pas seulement l'économie, mais la vie sociale dans son ensemble qui ne peut pas être régulée par une classe dominante, parce que les problèmes que pose une société moderne échappent au pouvoir de toute couche dirigeante, tant la gestion cohérente de la société est impossible si la " majorité des hommes est réduite au rôle d'exécutant, si leurs capacités d'organisation, d'initiative, de création sont systématiquement réprimées ${ }^{8}{ }^{\prime} »$.

Castoriadis a entrevu précocement et l'extension de la domination dans le capitalisme bureaucratique à tous les domaines de la vie sociale et la nouvelle géographie de la contestation sociale que cela entraînait. Cependant, l'apathie politique prolongée qu'il constate chez les travailleurs et les citoyens à partir des années 1960 l'amène à un diagnostic historique de plus en plus sombre. Il finira même par dire que l'impasse du projet d'autonomie dans la société contemporaine provient de la privatisation intégrale de la sphère publique.

Ne minimisons pas la raison historique qui rend compte de l'inflexion théorique repérable au niveau de la socio-ontologie chez Castoriadis. C'est le renversement d'un cycle historique d'émancipation révolutionnaire et l'hibernation du projet d'autonomie qui le poussent à une réflexion radicale sur l'autonomie. Sans abandonner tous les présupposés de la réalité-comme-production, Castoriadis se déplace progressivement, mais résolument, vers les raisons qui rendent possible, à tout moment, l'irruption de l'imaginaire instituant dans la société. Le déplacement est de taille. Le cœur de l'interrogation de Castoriadis va désormais porter (en fait, il est plus juste

81 Cornelius Castoriadis, L'expérience du mouvement ouvrier, t. I, Comment lutter, Paris, Union Générale d'Éditions, 1974, p. 249-277.

82 Cornelius Castoriadis, « Sur le contenu du socialisme, III » [1958], dans L'expérience du mouvement ouvrier, t. II, Paris, Union Générale d'Éditions, 1974, p. 71.

83 Cornelius Castoriadis, «Le mouvement révolutionnaire sous le capitalisme moderne » [1960-1961], dans Capitalisme moderne et révolution, t. II, Paris, Union Générale d'Éditions, 1979, p. 152. 
d'affirmer qu'il va alterner entre la production et la représentation) sur ce qui fait tenir ensemble une société, ce par quoi elle se dote d'un monde propre et qui, selon lui, ne peut être compris qu'à partir de la pratique auto-instituante. Disons-le clairement: malgré son oscillation, la ligne centrale de son œuvre se détourne plus ou moins sournoisement de l'ontologie de la production au sens fort du terme au profit d'une conception socio-ontologique élargie de la réalité comme représentation. La question de l'autonomie et de l'hétéronomie des formes instituées prend dès lors la place centrale dans l'analyse de Castoriadis, en faisant ainsi largement l'impasse sur une étude effective des résistances repérables au niveau de l'action - le propre justement d'une socio-ontologie de l'action.

En tout cas, la rupture avec Marx sera profonde et déterminante. Pour Castoriadis, si chez Marx coexistent à l'origine deux conceptions opposées, le déterminisme et la lutte des classes, il verra progressivement dans l'œuvre de Marx une réduction de la deuxième à la première. Du jeune Marx au Marx du Capital, la découverte de la véritable signification historique de la lutte des classes cède le pas à l'écriture d'un ouvrage d'économie politique d'où celle-ci est totalement absente. C'est dans le sillage de cette critique que Castoriadis pose sa découverte majeure: aucune théorie ne pourra jamais être exhaustive puisque l'activité humaine est toujours capable de faire constamment surgir du nouveau. Il soutient même avoir mis en lumière un élément caché de la pensée héritée et être arrivé ainsi à penser le mode d'être propre au social-historique - son être comme imaginaire social.

L'imaginaire, au sens que lui donne Castoriadis, est ce dans quoi se produisent les schèmes ou les figures qui sont les conditions dernières du représentable ou du pensable. Il s'agit d'une ouverture inaugurale que Castoriadis dénomme l'« imaginaire radical » tant il définit à la fois le surgissement du nouveau et une capacité d'exister « dans et par la position d'images ». De cet imaginaire radical, les deux principales manifestations étudiées par Castoriadis sont l'imagination radicale de la psyché et l'imaginaire social instituant au plan collectif. Une formation social-historique se constitue en s'instituant, c'est-à-dire en instaurant un magma de significations imaginaires sociales, valables pour tous les membres de la collectivité 84 . Les significations imaginaires sociales structurent donc les représentations du monde en général, elles désignent les finalités de l'action (en imposant ce qui est à faire et à ne pas faire) et enfin, elles établissent les affects d'une société (ce à quoi une société donne de la valeur). Ces formes sont créées par chaque société et font être au sens littéral un monde dans lequel cette société s'inscrit et se donne une place. L'emprise de la socio-ontologie de la représentation (dont atteste également la similitude des formules avec d'autres travaux ayant cette inspiration) est patente.

84 Cornelius Castoriadis, «Science moderne et interrogation philosophique » [1972], dans Les carrefours du labyrinthe, I, Paris, Seuil, 1978, p. 147-217. 
Soulignons l'essentiel : l'originalité de Castoriadis, au cœur de la socio-ontologie de la représentation, est d'affirmer (à l'encontre d'autres lectures) que le monde est prêt à accueillir n'importe quel type de signification imaginaire sociale. Ce n'est que sur cette réalité première que les sociétés bâtissent des institutions dont l'objectif explicite est de canaliser (comme c'est le cas avec le capitalisme bureaucratique) les relations entre les différents éléments. Castoriadis insistera maintes fois sur le rapport, hautement complexe, s'établissant entre le niveau «synchronique »d'une société (la dimension ensidique de la réalité, de nature proprement logique, où il est toujours possible de repérer des éléments, des classes, des propriétés, des relations) et le niveau "diachronique », le surplus imaginaire qui dépasse cette logique, et qui s'exprime en opposition, en contraste, voire en s'insérant partiellement en lui.

Pour Castoriadis, le mot « imaginaire » veut donc souligner l'aspect créateur du monde déployé par l'imagination de l'homme. Les éléments ne prennent sens qu'à l'intérieur d'un cadre de signification qui les excède et duquel ils tirent à proprement parler leur signification. Castoriadis souligne la nature ontologique du socialhistorique en tant que création continuelle, comme institution, comme monde de significations sociales imaginaires comprises comme primaires, inaugurales, irréductibles. L'imaginaire social est position, création, faire, être. Bien entendu, la "production» et l'«action» sont bel et bien présentes, mais elles ne le sont que cernées et définies à partir de la question de la représentation-comme-réalité. Rien n'en atteste mieux que le fait que ce sont les problèmes propres au regard socioontologique de la représentation qui deviennent vraiment actifs dans sa pensée, à commencer par la difficulté à appréhender les résistances effectives de la réalité, sinon sous la forme de "privatisations » des individus ou d' « hétéronomies » des collectifs, autant dire d' "aliénations » (un mot qui cache mal la difficulté à étudier les résistances de la réalité autrement que comme égarement représentationnel).

Ce déplacement matriciel vers la socio-ontologie de la représentation rend compte d'ailleurs de l'analyse historique fort critique que Castoriadis formule : les sociétés ont tendance à se cacher leur origine auto-instituée. La société est hétéronome, puisque le nomos, la loi, est supposée être donnée par quelqu'un d'autre. En fait, la création des institutions est imputée à une instance extra-sociale échappant au pouvoir d'action des hommes. Contre cette dérive, une société est autonome lorsqu'elle est ouverte à une interrogation illimitée, lorsqu'elle a à faire l'institution explicite globale de ses institutions, surtout lorsqu'elle sait qu'elle fait ses lois et lorsqu'elle est capable de les mettre explicitement en cause ${ }^{85}$. La "production » semble ainsi avoir

85 La redécouverte de la société comme auto-instituée passe par le rachat de la conception primordiale de la praxis. " Nous appelons praxis ce faire dans lequel l'autre ou les autres sont visés comme êtres autonomes et considérés comme l'agent essentiel du développement de leur propre autonomie » (Cornelius Castoriadis, L'institution imaginaire de la société, Paris, Seuil, 1975, p. 103). L'autonomie de la praxis désigne donc une nouveauté radicale, puisqu'elle n'est jamais l'application stricte d'un savoir préalable. Dans ce sens, la praxis n'est pas le faire humain dans sa totalité, mais n'est qu'une modalité du faire humain, celui qui vise une certaine transformation de son objet, c'est-à-dire, la pratique qui 
toujours une fonction déterminante, mais à bien y regarder, ce sont toujours les «significations» qui structurent son regard socio-ontologique - les résistances « hétéronomes » de la réalité sont conçues comme étant solubles dans des représentations «autonomes». Autant dire que Castoriadis n'aborde pas, ou guère, les contraintes effectives de la réalité sur l'action. Cette impasse est surtout patente lorsqu'on prend acte que Castoriadis défend une conception radicale de la création davantage associée à l'imagination radicale et à l'inconscient que véritablement à l'action. C'est au final dans la psyché singulière que se trouve la garantie ultime et permanente de la possibilité de création irréductible dans l'histoire86.

Comprenons bien ce point. Les significations imaginaires sociales préexistent à l'individu, elles sont toujours déjà-là. L'irréductibilité de l'institution à un agir individuel contraint justement à accepter, selon Castoriadis, l'existence d'un niveau d'être inconnu de l'ontologie héritée, le social-historique comme collectif anonyme, instituant et créateur de significations. Le lien entre le social-historique et les individus est un type de relation inédit et original, impossible à penser sous les catégories du tout et des parties, de l'ensemble et de ses éléments, de l'universel et du particulier. Il s'agit de deux pôles irréductibles : la société fait les individus qui font la société87. La sociologie avait, par ailleurs, affirmé cela de maintes manières auparavant. Mais chez Castoriadis, ce qui reste opaque au cœur de cette dialectique est étrangement la réalité ontologique de la société, définie comme "ce qui résiste » - une dimension jamais clairement élucidée. La tension d'ailleurs est bien présente dans l'ambiguïté observable dans l'utilisation de la notion d'imaginaire. Elle renvoie à la fois aux significations communes à partir desquelles l'individu se déploie dans le monde (celles à partir desquelles un collectif d'hommes se dote littéralement d'un monde) et à la capacité créatrice des sociétés et des hommes. Le tour de passe-passe de Castoriadis consiste à établir l'irréductibilité de ces deux pôles par le constat de deux déficits : d'un côté, il affirme que l'imagination radicale plonge dans des significations collectives qui l'excèdent par nature et de l'autre côté, il extrapole de cet état de fait, une supposée créativité collective qu'il ne décrit cependant que dans les termes de l'imagination radicale (l'inconscient). Il n'y a pas tant réduction de l'un à l'autre que déplacement - et compréhension - du mode de constitution et d'opération de l'un (l'inconscient) sur l'autre (le social-historique). Il n'y a pas alors à vraiment parler deux grandes manifestations de l'imaginaire radical, individuelle et collective, mais une seule source, l'imagination radicale à l'œuvre dans l'inconscient, et le déplacement intellectuel de son mode de constitution, voire d'être, à l'imaginaire so-

considère l'autre comme autonome et qui tente en même temps de l'aider à accomplir son autonomie. On comprend alors l'importance majeure que Castoriadis octroie aux trois activités pratico-poïétiques que sont la pédagogie, la psychanalyse et la politique (Cornelius Castoriadis, « Psychanalyse et politique » [1989], Les carrefours du labyrinthe, IV. Le monde morcelé, Paris, Seuil, 1996, p. 141-154).

86 Cornelius Castoriadis, L'institution imaginaire de la société, op. cit., p. 431.

87 Cornelius Castoriadis, « Pouvoir, politique, autonomie » [1989], Les carrefours du labyrinthe, III. Le monde morcelé, Paris, Seuil, 1990, p. 114. 
cial ${ }^{88}$. Mais par ce truchement, son œuvre fait systématiquement l'impasse sur les problèmes pratiques de la "création », autant dire sur les résistances effectives de la réalité, telles qu'elles sont analysées par les socio-ontologies de l'action.

En fait, le projet d'une socio-ontologie totale autour de l'imaginaire révèle la principale tension constitutive de son œuvre, à savoir qu'il y a au fond deux sources de l'autonomie et de la création chez Castoriadis. La première, de nature plus sociale et historique, trouve son expression initiale, comme on l'a évoqué, dans les luttes ouvrières, dans la réalité irrépressible de l'implication humaine au travail. Cette créativité est d'une nature proprement sociale - et aurait dî engager Castoriadis dans la voie explicite d'une socio-ontologie de l'action afin d'analyser en aval et en amont les résistances effectives de la réalité. Cependant, et à la suite d'un diagnostic historique de plus en plus sombre sur la société contemporaine, Castoriadis s'est progressivement focalisé de manière exclusive sur une socio-ontologie de la représentation (l'imaginaire radical). Or, cette deuxième conception de la " création » est de nature avant tout psychique. Ou plutôt, c'est dans la réalité de l'inconscient psychique qu'elle s'exprime le mieux, là où on trouve les éléments allant dans le sens d'une création radicale et irrépressible. En ancrant la possibilité d'un imaginaire radical au niveau de l'inconscient, Castoriadis a sans doute laissé «ouvert» et indéterminé le domaine de la création; mais ce «sauvetage" théorique n'a été fait qu'au prix de l'abandon de l'étude concrète des contraintes effectives et historiques de la réalité.

Chez Castoriadis, le passage d'une ontologie de la production vers une ontologie de la représentation s'est donc fait en court-circuitant l'ontologie de l'action. Répétons-le, au moment du tournant central de son œuvre, deux ouvertures étaient possibles. D'une part, la socio-ontologie de la production chère au "premier» Castoriadis, ancrée dans une réalité conflictuelle irrépressible, celle de la lutte des classes, et qui aurait pu - dû - l'engager vers une socio-ontologie explicite de l'action. D'autre part, et c'est la voie largement suivie par le «deuxième » Castoriadis, l'enfermement dans une socio-ontologie de la représentation, des significations sociales, du radicalement autre, des capacités irrépressibles de création humaine. La première perspective aurait dû permettre à Castoriadis d'aborder la nature et la force des résistances effectives inscrites dans la "matière » sociale. En glissant vers la seconde perspective, Castoriadis n'a fait que retrouver l'opposition, assez classique, entre d'un côté la forte cohérence synchronique repérable entre tous les éléments d'une culture à un moment donné (le fait justement, qu'ils finissent par structurer un monde au sein duquel une forte homologie s'établit entre les individus et leur société) et de l'autre côté, et dans une dimension diachronique, l'appel quelque peu incantatoire à une capacité irrépressible de création radicale dans l'histoire.

88 Pour un développement détaillé de ce raisonnement, $c f$. Danilo Martuccelli, « Cornelius Castoriadis : promesses et problèmes de la création », Cahiers internationaux de sociologie, vol. 113, 2002, p. 285 305. 
Après un long détour, Castoriadis redécouvre l'antinomie kantienne du déterminisme du monde et de la liberté (création) du sujet.

\section{Conclusion}

La réflexion socio-ontologique aura donc emprunté trois grandes voies. Si les trois perspectives présentées ne définissent pas tout l'éventail des démarches possibles, elles ont toutes cependant une indéniable centralité dans la sociologie. Chaque fois, en partant d'un noyau dur, il est question d'aborder les résistances de la réalité à partir de différentes problématisations. En premier lieu, à partir de l'agencement des représentations, de leur mise en trame institutionnelle, de leur capacité ainsi à fairemonde. Ensuite par le truchement d'une réalité qui, conçue comme étant globalement fabriquée par les individus, donne lieu à des résistances multiples au fur et à mesure que les objectivations produites s'autonomisent de leurs auteurs. Enfin, en considérant, lors du déroulement des actions, les heurts pratiques effectifs en aval ou les limites imaginaires en amont en tant que double butoir des conduites. Ces perspectives ne sont pas forcément excluantes, mais elles n'en engagent pas moins des problématisations fort différentes.

Dans l'espace de ces débats socio-ontologiques, l'œuvre de Castoriadis est exemplaire pour deux grandes raisons. D'une part, parce que, tout en visant plus ou moins implicitement un agencement particulier entre les différentes ontologies, il a cependant finalement proposé, presque inéluctablement, une socio-ontologie unilatérale de l'imaginaire autour du modèle de la représentation. D'autre part, et cette fois-ci à partir d'un regard critique, parce que son œuvre montre la principale difficulté de bien des socio-ontologies contemporaines - l'incapacité à rendre compte précisément de la nature et de la force effectives des résistances de la réalité. Cette seconde difficulté, étant donnée l'envergure de la pensée de Castoriadis, est une véritable leçon : si la problématisation centrale et la plus heuristique des regards socio-ontologiques concerne - tôt ou tard - la nature et la force des résistances que le monde oppose aux acteurs, c'est du côté des questionnements ouverts sur les liens entre l'ontologie et l'action que résident les principales promesses analytiques à venir. 